\title{
Ruptured renal artery aneurysm in pregnancy
}

\author{
N Mdlalose, ${ }^{1}$ MB ChB; T L Khumalo, ${ }^{2}$ MB ChB, FCOG (SA), MMed (O\&G) \\ ${ }^{1}$ Department of Obstetrics and Gynaecology, Pietermaritzburg Metropolitan Hospital Complex, University of KwaZulu-Natal, Durban, \\ South Africa \\ ${ }^{2}$ Department of Obstetrics and Gynaecology, Edendale Regional Hospital, University of KwaZulu-Natal, Durban, South Africa
}

Corresponding author: N Mdlalose (mdlalosen88@gmail.com)

Renal artery aneurysm is a rare condition, the incidence being $\sim 0.01-1 \%$. In pregnancy this condition is rare but carries a significant mortality. Predisposing factors in pregnancy are thought to be physiological, hormonal and haemodynamic. Clinical diagnosis requires a high level of suspicion, as patients are usually asymptomatic prior to rupture. The clinical presentation often mimics other common obstetric conditions such as labour, uterine rupture or placental abruption. It is essential at laparotomy in cases of intra-abdominal bleeding that a thorough search is made for the source of blood loss when no obvious obstetric cause is found. We report a case of a maternal death that was due to rupture of a renal artery aneurysm in the immediate peripartum period in a patient with chronic hypertension.

S Afr J Obstet Gynaecol 2020;26(2):76-78. https://doi.org/10.7196/SAJOG.2020.v26i2.1552

\section{Case report}

A 43-year-old (gravida 3, parity 1) initiated antenatal care at a community health centre at 12 weeks' gestation. She was HIVpositive and virally suppressed on a fixed-dose regimen. Her booking blood pressure (BP) was 140/90 $\mathrm{mmHg}$ with no abnormalities on urinalysis. She had no history of a previous hypertensive disorder in pregnancy; she had one previous caesarean delivery (CD) in 1998 for an unknown indication and one previous first-trimester miscarriage. Her antenatal records noted a history of chronic hypertension, which was diagnosed before the age of 35 and managed with antihypertensive agents. This index pregnancy was with a new sexual consort, unplanned, however accepted, and she had a total of ten antenatal visits. She was attended to at a high-risk antenatal care level from 18 weeks' gestation. Her blood pressure remained well controlled on methyldopa and she had no evidence of superimposed pre-eclampsia.

Her acute presentation to Edendale Regional Hospital in KwaZuluNatal Province, South Africa, was with a complaint of labourlike pains at 38 weeks. On examination she was found to be haemodynamically stable, with a well-controlled BP and with no evidence of superimposed pre-eclampsia. She was found not to be in labour. However, a non-stress test (NST) showed pathological features, notably late decelerations.

The patient was counselled and consented to emergency CD for suspected fetal compromise and a bilateral tubal ligation as per her antenatal plan. Her pre-operative haemoglobin was $11 \mathrm{~g} / \mathrm{dL}$. The $\mathrm{CD}$ was performed under spinal anaesthesia and was noted to be uneventful, without evidence of placental abruption or postpartum haemorrhage (PPH). The baby weighed $2600 \mathrm{~g}$ and had a 5-minute Apgar score of $9 / 10$ with a normal cord $\mathrm{pH}$ gas sample. During the theatre recovery period the maternal vital signs were recorded as normal, the uterus was well contracted and a normal minimal lochia rubra was observed. The patient was then transferred to the postnatal ward for further postoperative observations and care.
Approximately 7 hours post delivery, the attending midwife noted that the patient was sweating and pale with cold and clammy extremities. The vital signs were as follows: $\mathrm{BP}$ was $73 / 48 \mathrm{mmHg}$, pulse 138 beats/minute and the oxygen saturation was $97 \%$ on room air. At this point, she had no active vaginal bleeding and had mild distension of the abdomen with the uterus difficult to palpate. An assessment of hypovolaemic shock was made, immediate resuscitation was commenced, and intravenous (IV) fluids were commenced, one packed red cell concentrate (RCC) transfused and tranexamic acid (Cyklokapron) $1.5 \mathrm{~g}$ IV administered.

The patient responded positively to fluid resuscitation, with an improved mean arterial BP (MAP) of 70 and she was subsequently transferred to the obstetric high care unit (HCU) for ongoing resuscitation and to await an exploratory laparotomy. An arterial blood gas analysis revealed a compensated metabolic acidosis. There was a normal clotting profile and the HCU admission haemoglobin was $8.1 \mathrm{~g} / \mathrm{dL}$.

The patient underwent an emergency exploratory laparotomy, which was performed via the previous transverse abdominal wall incision. The essential findings were $800 \mathrm{ml}$ of clotted haemoperitoneum, the uterine surgical site had secure haemostasis, the uterus was well contracted and no broad ligament or pelvic haematomas were noted at this stage. Further exploration of the abdominal cavity was negative, with no visible source of active haemorrhage. The abdomen was washed out, closed and the patient transferred back to HCU for further monitoring. In HCU the patient had a persistent tachycardia, the haemoglobin remained static at $8 \mathrm{~g} / \mathrm{dL}$ and BP was within normal limits. Her urine output was $1.1 \mathrm{ml} / \mathrm{kg} / \mathrm{h}$, the fluid maintenance was continued at $80 \mathrm{ml} / \mathrm{h}$ and one unit of RCC ordered.

Approximately 6 hours post laparotomy, the patient had a generalised tonic seizure, her Glasgow Coma Scale dropped to 3 and she became bradycardic. She was found to have further increased abdominal distension and significantly marked pallor. 
She had circulatory failure and cardiac arrest that required active cardiopulmonary resuscitation and tracheal intubation in HCU. After return of spontaneous circulation was achieved, she was taken back for re-laparotomy via midline incision. The intra-operative findings were as follows: a large right retroperitoneal haematoma extending from the right renal pelvis to the right paracolic gutter; the pelvic organs and previous surgical sites were as previously noted.

Multidisciplinary assistance was provided by general and trauma surgeons. Further surgical exploration revealed a large right peri-renal haematoma, at the root of which was a ruptured right renal artery aneurysm. The aneurysm was resected, the artery ligated, followed by a right nephrectomy as a salvage manoeuvre, and haemostasis was achieved. Posthumously the histopathology taken from the renal artery confirmed features compatible with a renal artery wall aneurysm.

In view of disseminated coagulopathy (DIC) the abdomen was packed for planned review in 48 hours for washout and closure. The patient was transferred to the intensive care unit (ICU) for ongoing peri-operative physiological support and further resuscitation. She required inotropic support and correction of DIC as she displayed a prolonged $\mathrm{R}$ phase on the thromboelastogram (TEG). She was noted to have recurring coagulopathy and acute kidney injury and demised in ICU despite active treatment and care.

\section{Discussion}

We report a case of rupture of the renal artery aneurysm (RRAA) in the peripartum period in a patient with a background of chronic hypertension, who may plausibly have been an under-investigated young hypertensive. As part of her antenatal investigations, being managed as a chronic hypertensive, our patient had a normal echocardiogram, normal renal function, and a normal grey-scale renal sonogram and no evidence of chronic renal disease. Her renal ultrasound report is shown in Fig. 1.

\section{STUDY NO: 2019-6240 PATIENT}

\section{ULTRASOUND OF THE KIDNEY}

Kidneys are normal in size and echopattern.

Right $=10.3 \mathrm{~cm}$ and Left $=10.2 \mathrm{~cm}$

There is good cortico-medullary differentiation.

No focal parenchymal lesion.

No hydronephrosis or hydroueter.

\section{CONCLUSION:}

- No obvious pathology noted.

\section{DATE: $14 / 10 / 2019$ \\ DATE: $14 / 10 / 2019$ \\ TIME: 08:46}

Fig. 1. Patient's renal ultrasound report.
The suspicion of an RRAA was low as typically, she was asymptomatic during pregnancy. A retrospective collateral history from the husband revealed that she had complained of feeling dizzy and had sharp pains in her right flank for 2 weeks prior to presentation.

Renal artery aneurysm is a rare condition with an incidence of $0.01-1 \%$ but carries a very high mortality rate for the mother and fetus. $^{[1-3]}$ Data regarding RRAA in the postpartum period are limited to case reports. Hwang et al. ${ }^{[4]}$ reported two cases that were diagnosed post partum and managed successfully. The increase in the blood flow, abdominal pressure and steroid production in the presence of an aneurysm of $>2 \mathrm{~cm}$ have been postulated as contributors to the increased risk of rupture during and immediately after pregnancy. ${ }^{[4]}$ Renal artery aneurysm results from dilatation due to weakness of the intima and media layers of the artery. The causes can be congenital or acquired, including degenerative conditions like atherosclerosis and in some rare cases malignancies.

There are hypotheses that RRAA during pregnancy is mainly due to congenital abnormalities. These congenital abnormalities result in a weakened arterial wall or malformation. Both hypertension and pregnancy increase the risk of rupture of the aneurysm..$^{[5]}$ The diagnosis of RRAA in pregnancy is likely to be an incidental finding during surgery for other non-related conditions, or during exploration in patients that have haemodynamically decompensated following surgery or trauma.

Our patient presented with clinical signs suspicious of labour and did not have the typical findings of right flank pain and haematuria, and urinalysis on admission was normal. However, retrospectively there was a history of flank pain. The remarkably acute and short interval of the patient's decompensation following the first laparotomy correlates with the significance of the morbidity and mortality associated with rupture of an undiagnosed renal artery aneurysm. Love et al. ${ }^{[6]}$ report that RRAA during pregnancy can cause severe distortion of the retroperitoneal anatomy and make it difficult to palpate the opposite kidney. In our case the left kidney was normally situated.

Management of an RRAA during pregnancy may involve renal artery reconstruction and embolisation where there is acute haemorrhage. Further management may include stenting, coiling and salvaging procedures of the kidney. In complicated cases with severe renal injury, nephrectomy may be recommended as a salvage procedure, where haemorrhage may be at life-threatening levels.

This case highlights that prudent management of chronic hypertension in pregnancy warrants thorough history taking, examination and investigation for target organ affectation. A previous history of early-onset pre-eclampsia ought to be ruled out. In the case of a chronic hypertensive patient, the occurrence of hypovolaemic shock following an uneventful CD should trigger suspicion of among others the possibility of an RRAA. The gold standard should be thorough abdominal and retroperitoneal exploration via a midline incision, preferably by a multidisciplinary team whenever no obvious source of haemorrhage can be identified. Obstetricians naturally search for obstetric causes. When none is found, a further search for the unusual is mandatory, and should ideally be performed by a general surgeon. A midline incision is expedient and allows complete access into the entire abdominal cavity.

\section{Conclusion}

Renal artery aneurysm is a rare condition, with rupture likely to happen in pregnancy as a result of haemodynamic and hormonal changes. There is a paucity of data on this subject, with most of 


\section{CASE REPORT}

the understanding arising mainly from case reports. Routine renal angiograms are not recommended in pregnancy, except in cases of early-onset pre-eclampsia, where they may play a role. Renal artery aneurysm during pregnancy remains difficult to diagnose, requiring a high index of suspicion.

Acknowledgement. Professor Emeritus J Moodley for review of the manuscript. Author contributions. The authors collaborated in the drafting of the case summary; the conceptualisation of the discussion in line with the literature review was supervised by TLK. Pre-submission editing was a joint effort by both authors.

Funding: None.

Conflict of interest: None.
1. Schorn B, Falk V, Dalichau H, Mohr FW. Kidney salvage in a case of ruptured renal artery aneurysm. J Cardiovasc Surg 1997;5(1):134-136. https://doi.org/10.1016/S0967-2109(95)00041-0

2. Cohen JR, Shamash FS. Ruptured renal artery aneurysm during pregnancy. J Vasc Surg 1987;6(1):51-59. https://doi.org/10.1067/mva.1987.avs0060051

3. Soliman KB, Shawky Y, Abbas MM. Ruptured renal artery aneurysm during pregnancy: A clinical dilemma. BMC Urol 2006;6(22):1-4. https://doi.org/10.1186/1471-2490-6-22

4. Hwang PF, Rice DC, Patel SV. Successful management of renal artery aneurysm post caesarean section. J Vasc Surg 2011;54(2):519-521. https://doi.org/10.1016/j.jvs.2010.12.041

5. Hack RW. Rupture of the left renal artery aneurysm during pregnancy Am J Obstet Gynecol 1953;65(5):1142-1145. https://doi.org/10.1016/0002-9378(53)90649-8Love

6. Love WK, Robinette MA, Vernon CP. Renal artery aneurysm in pregnancy. J Vasc Surg 1981;126(6):809-911. https://doi.org/10.1016/s0022-5347(17)54759-x.

Accepted 20 August 2020 